\title{
Eine Ausstellung und viele Fragen
}

\section{Erhard Taverna}

«Verstehen Sie doch endlich dort am lateinischen Meer, dass es sich bei den Vorgängen in Deutschland [...] um das Hervortreten eines neuen biologischen Typs [handelt], die Geschichte mutiert und ein Volk will züchten. Wollen Sie, Amateur der Zivilisation und Troubadour des westlichen Fortschritts, endlich doch verstehen, es handelt sich gar nicht um Regierungsformen, sondern um eine alte, vielleicht um die letzte grossartige Konzeption der weissen Rasse, wahrscheinlich um eine der grossartigsten Realisationen des Weltgeistes überhaupt [...].»

Gottfried Benn, der Arzt-Schriftsteller, verteidigte seine Haltung in einem Brief an Klaus Mann, geschrieben 1933. Wenig später verabschiedete er sich von der neuen Politik und suchte als Militärarzt Zuflucht in der «inneren» Emigration. Die überwältigende Mehrheit seiner Kollegen folgte, überzeugt von einer langen Tradition rassenhygienischer Theorien, der «grossartigen Konzeption». Die Wanderausstellung des «United States Holocaust Memorial Museum» mit dem Titel «Tödliche Medizin» bot in Dresden bis Ende Juni 2007 eine unverzichtbare Gelegenheit, das böse Thema zu vertiefen. Einzigartig wegen der Fülle an ausgestellten Dokumenten, bemerkenswert aber auch wegen der Absicht einer kritischen Auseinandersetzung mit aktuellen Utopien einer biologischen Verbesserung des Menschen. Schritt für Schritt wurde der lange Weg bis hin zur nationalsozialistischen «Endlösung» nachgezeichnet. Den Ausdruck «Eugenik» prägte der britische Wissenschaftler Francis Galton (1822-1911), ein Vetter von Charles Darwin: «[...] what a galaxy of genius might we not create!» Seine Ansichten der Zuchtwahl waren international weit verbreitet, erinnert sei an den Schweizer Psychiater Auguste Forel (1848-1931), der sich erfolgreich für die Zwangssterilisation einsetzte. Forscher wie der Kriminalist Cesare Lombroso in Italien entwickelten eine Anthropologie unwerten Lebens, die zu Sterilisationsgesetzen in den USA, Kanada und der Schweiz führte. Schon lange vor dem Dritten

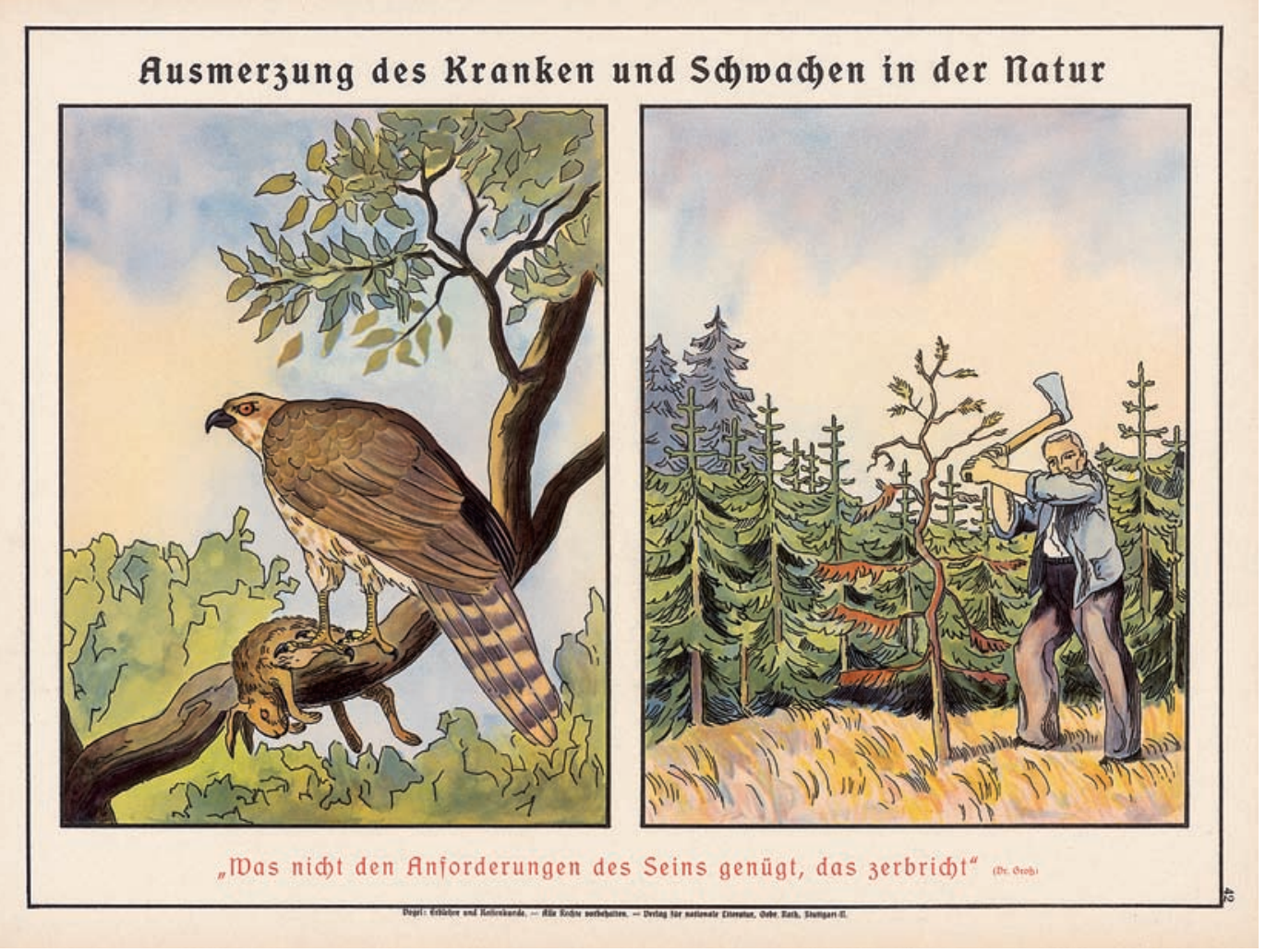

«Ausmerzung des Kranken und Schwachen in der Natur». Lehrtafel aus «Erblehre, Abstammungs- und Rassenkunde in bildlicher Darstellung» von Alfred Vogel, 1938. (c) Deutsche Nationalbibliothek Leipzig. 
Reich waren die Grundzüge einer Rassenhygiene ausformuliert. Einmal in Gang gesetzt, erfassten die Indikationen immer weitere Gruppen, erst Geisteskranke und Alkoholiker, dann Kriminelle, sozial Randständige, Zigeuner, Vaganten, Homosexuelle und schliesslich sogenannte «Reinlandbastarde», Kinder aus Verbindungen deutscher Frauen mit Marokkanern oder Vietnamesen der französischen Besatzungstruppen. Weltweit warnten Mediziner und Forscher vor der abnehmenden Geburtenrate gesunder Mittelstandsfamilien, bei gleichzeitig zunehmender Zahl von Kranken, Schwachen und Erwerbsunfähigen. Humangenetiker vieler Länder waren überzeugt, dass moderne Medizin und staatliche Wohlfahrt die «natürliche Auslese» behinderten und diese zugunsten tüchtiger, intelligenter und arbeitsfähiger Bürger und Bürgerinnen durch drastische Eingriffe zu korrigieren sei. Eine wachsende Zahl von Experten vermengte eugenische mit rassischen Vorstellungen, die vor allem in der Rassenvermischung ein bedrohliches Element entdeckten. Der Internist Alfred Ploetz prägte das Wort «Rassenhygiene» und war 1905 Herausgeber der weltweit ersten Zeitschrift «Archiv für Rassenund Gesellschafts-Biologie». Die Massnahmen des Dritten Reiches zur völkischen Gesundung wurden von Forschern und Medizinern legitimiert und mitgetragen. Einzige Ausnahme waren die rund 8000 jüdischen Ärzte (11\% aller Medizinern) denen die Kassenzulassung entzogen wurde. Der Gang durch die Ausstellung glich in weiten Teilen einer Porträtgalerie prominenter Fachärzte, die aktiv als Berater, Gutachter, Lehrer, Gesetzgeber und Vollstrecker an allen Schaltstellen der Macht den Vollzug energisch vorantrieben. Der weitere Verlauf ist bekannt: Der Sterilisation und Abtreibung folgte die Euthanasie unwerten Lebens: psychiatrisch Kranker, Behinderter und missgestalteter Neugeborener. Es war die Generalprobe zum Massenmord an Juden, russischen Kriegsgefangenen und Zigeunern.

Die Katastrophe hat alle Gewissheiten zerstört. Unverständnis und Verdrängen bedrohen unsere kulturelle Existenz. Was ist Wissenschaft? Wird sie immer ein Werkzeug der Macht sein? Ist sie kontrollierbar? Was braucht es, damit sie human bleibt? Warum hat es Jahrzehnte gedauert, bis das Problem erkannt wurde? Und warum konnten die meisten der beteiligten Ärzte nach 1945 ungehindert weiterarbeiten und Karriere machen? Die meisten Anklagen gegen fehl-

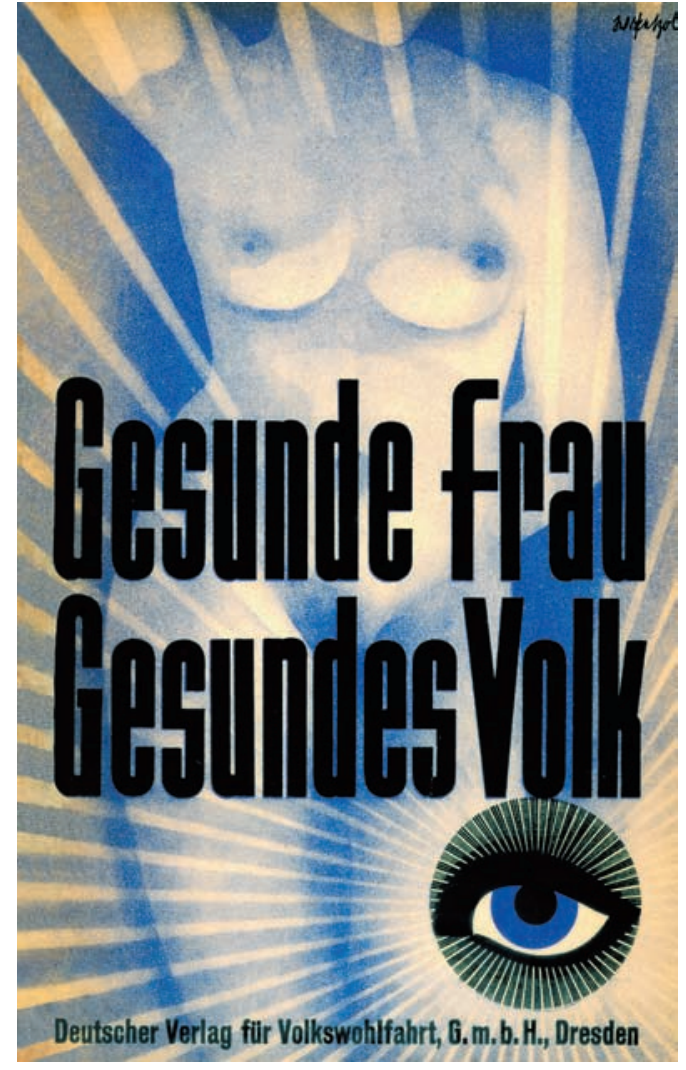

Titelseite der Broschüre zur Ausstellung «Gesunde Frau Gesundes Volk» (1932) im Deutschen Hygiene-Museum. (c) Deutsches Hygiene-Museum, Dresden.

bare Kollegen hatten in den Nachkriegsjahren keine juristischen Folgen, sie wurden freigesprochen oder die Prozesse endlos verschleppt. In den Nürnberger Prozessen wurden einige wenige stellvertretend als Sündenböcke verurteilt. Der die medizinischen Prozessakten zusammenfassende Bericht des jungen Psychiaters Mitscherlich wurde von den Ärztegesellschaften der vier Besatzungszonen weitgehend ignoriert, er selbst verleumdet, die Verbreitung aktiv hintertrieben. Noch Anfang der 60er Jahre wurden Kritiker als Nestbeschmutzer diffamiert. Die Ausstellung zeigt nur Fakten, sie kommentiert nicht, sie provoziert verstörende Fragen. Der umfassende, auch buchtechnisch hervorragende Ausstellungskatalog «Deadly Medicine. Creating the Master Race» war nur englisch erhältlich. Gedruckt wurde er in China. Gottfried Benn hat in den Trümmern Berlins wieder als Dermatologe gearbeitet. Auf seinen Brief ist er nie mehr zurückgekommen. 